\title{
Arabidopsis PDK1: identification of sites important for activity and downstream phosphorylation of S6 kinase
}

\author{
Lotta Otterhag $^{\text {a,*, Niklas Gustavsson }}{ }^{\mathrm{b}}$, Magnus Alsterfjord ${ }^{\mathrm{a}}$, Christophe Pical ${ }^{\mathrm{a}}$, \\ Hans Lehrach ${ }^{\mathrm{b}}$, Johan Gobom ${ }^{\mathrm{b}}$, Marianne Sommarin ${ }^{\mathrm{a}}$ \\ ${ }^{a}$ Department of Plant Biochemistry, Lund University, P.O. Box 124, SE-22100 Lund, Sweden \\ b Mass Spectrometry Group, Max Planck Institute for Molecular Genetics, Ihnestr. 73, D-14195 Berlin, Germany
}

Received 19 November 2004; accepted 21 July 2005

Available online 18 August 2005

\begin{abstract}
The Arabidopsis thaliana protein kinase AtPDK1 was identified as a homologue of the mammalian 3-phosphoinositide-dependent protein kinase-1 (PDK1), which is involved in a number of physiological processes including cell growth and proliferation. We now show that AtPDK1, expressed in E. coli as a recombinant protein, undergoes autophosphorylation at several sites. Using mass spectrometry, three phosphorylated amino acid residues, Ser-177, Ser-276 and Ser-382, were identified, followed by mutational analyses to reveal their roles. These residues are not conserved in mammalian PDK1s. Mutation of Ser-276 in AtPDK1 to alanine resulted in an enzyme with no detectable autophosphorylation. Autophosphorylation was significantly reduced in the Ser177Ala mutant but was only slightly reduced in the Ser382Ala mutant. Other identified sites of importance for autophosphorylation and/or activity of AtPDK1 were Asp-167, Thr-176, and Thr-211. Sites in the mammalian PDK1 corresponding to Asp-167 and Thr-211 are essential for PDK1 autophosphorylation and activity. Autophosphorylation was absent in the Asp167Ala mutant while the Thr176Ala and The211Ala mutants exhibited very low but detectable autophosphorylation, pointing to both similarity and difference between mammalian and plant enzymes. We also demonstrate that AtS6k2, an A. thaliana homologue to the mammalian S6 kinases, is an in vitro target of AtPDK1. Our data clearly show that Asp-167, Thr-176, Ser-177, Thr-211, and Ser-276 in AtPDK1 are important for the downstream phosphorylation of AtS6k2. The results confirm that AtPDK1, like mammalian PDK1, needs phosphorylation at several sites for full downstream phosphorylation activity. Finally, we investigated A. thaliana 14-3-3 proteins as potential AtPDK1 regulatory proteins and the effect of phospholipids on the AtPDK1 activity. Nine of the 12 14-3-3 isoforms tested enhanced AtPDK1 activity whereas one isoform suppressed the activity. No significant effects on AtPDK1 activity by the various phospholipids (including phosphoinositides) were evident.
\end{abstract}

(C) 2005 Elsevier SAS. All rights reserved.

Keywords: Autophosphorylation; AtS6k2; 14-3-3 Protein; AGC kinase; Arabidopsis thaliana

\section{Introduction}

The 3-phosphoinositide-dependent protein kinase-1 (PDK1) plays a central role in mammalian systems by phosphorylating a number of different targets belonging to the AGC subfamily of protein kinases, of which PDK1 itself is also a member [1]. These targets, which include protein kinase $\mathrm{B}$ (PKB) isoforms, serum and glucocorticoid-inducible kinase (SGK), protein kinase C isoforms, p90 S6 kinase (S6K) and $\mathrm{p} 70$ ribosomal $\mathrm{S} 6 \mathrm{~K}$, are involved in various physiological pro-

\footnotetext{
* Corresponding author. Tel.: +46 46222 9382; fax: +46 462224116.

E-mail address: lotta.otterhag@plantbio.lu.se (L. Otterhag).
}

cesses, including cell proliferation, differentiation and apoptosis [1-3]. PDK1 homologues have been identified in Arabidopsis thaliana and rice (Oryza sativa), but the PDK1 signalling pathways in plants are largely unknown. The PDK1 from A. thaliana, AtPDK1, can complement a yeast mutant lacking the PDK1 homologues Pkh1 and Pkh2, as well as activate mammalian PKB in vitro [4]. However, very little is known about the natural targets of plant PDK1. A number of $A$. thaliana protein kinases have been suggested as potential PDK1 substrates [5] and recently two members of the AGC protein kinase family, AGC-1 and AGC-2, were shown to interact with AtPDK1 [6]. AGC-2, was demonstrated to be directly phosphorylated by AtPDK1 in a signalling pathway 
that is regulated by phosphatidic acid and involved in root hair growth [6].

Two plant homologues of the S6Ks, AtS6k1 and AtS6k2, have also been proposed to act as PDK1 targets [4]. These protein kinases, whose gene expression is enhanced in response to salt and low temperature stress, share $87 \%$ amino acid sequence similarity with the human S6K [7]. In addition, three phosphorylation sites that are critical for mammalian S6K activation, Thr-229, Ser-371 and Thr-389 are conserved in AtS6k1 and AtS6k2 [8]. In mammalian cells, the phosphorylation and activation of S6K by PDK1 promotes phosphorylation of the 40S ribosomal protein S6, which in turn controls cell growth and proliferation via the consequential regulation of $5^{\prime}$-terminal oligopyrimidine tract mRNA translation [2]. Interestingly, one of the A. thaliana S6K homologues, AtS6k2, has been shown to phosphorylate a plant ribosomal S6 protein in vivo, a phosphorylation sensitive to wortmannin and LY294002, PtdIns 3-kinase inhibitors that suppress mammalian S6K activity [8-10]. Also in maize (Zea mays), an S6K homologue, ZmS6K, phosphorylated a ribosomal S6 protein in vivo [11]. This indicates that activation of the plant ribosomal S6 protein is mediated by a signalling pathway similar to that of the mammalian S6K.

Mammalian PDK1 undergoes autophosphorylation via both cis (intramolecular) and trans (intermolecular) mechanisms, and several autophosphorylation sites have been identified [12-15]. Autophosphorylation of Ser-241 in human PDK1 is essential for its activity and the corresponding residue in mouse PDK1 was recently shown to be autophosphorylated via a trans mechanism dependent on PDK1 dimerisation [12,13]. Apart from PDK1 itself, no serine/threonine kinases able to phosphorylate PDK1 have been identified. However, Src, Fyn, and Abl tyrosine kinases have been shown to phosphorylate PDK1 in vitro and overexpression of v-Src in mammalian cells results in tyrosine phosphorylation and activation of PDK1 [15-17]. In addition, mammalian PDK1 is affected by interactions with PDK1-associating proteins, including PKC-related kinase 1 (PRK1), and Hsp90 [18-21], suggesting that its activity is controlled by interactions with other proteins as well. Furthermore, human PDK1 has been shown to interact with 14-3-3 $\theta$ and $\eta$ and a 14-3-3 recognition site has been identified at Ser-241 [22]. The human 14-3-3 $\theta$ negatively regulates PDK1 and thereby reduces the kinase activity to about half of its original activity [22]. The 14-33 proteins are $30 \mathrm{kDa}$ soluble proteins found in all eukaryotic organisms investigated so far and are involved in a multitude of physiological processes including signal transduction, regulation of transcription and enzymatic activity [23].

Very little is known about the characteristics of plant PDKs and of their downstream targets. In the present study, we show that the plant S6 kinase AtS6k2 acts as a substrate for AtPDK1 in vitro. Furthermore, we identify three autophosphorylation sites in AtPDK1, Ser-177, Ser-276 and Ser-382, and show that, in particular, phosphorylation of Ser-276 is essential for AtPDK1 downstream activity. Other sites identified that are of importance for AtPDK1 activity are Asp-
167, Thr-176 and Thr-211. We demonstrate that nine $A$. thaliana 14-3-3 isoforms positively regulate AtPDK1 autophosphorylation and activation of AtS6k2, whereas one A. thaliana 14-3-3 isoform, o, negatively regulates the kinase activity.

\section{Materials and methods}

\subsection{Expression and purification of recombinant proteins}

AtPDK1 and AtS6k2 cDNAs, kindly provided by M. Deak (Dept. of Biochemistry, University of Dundee, UK) and K. Shinozaki (RIKEN, Ibaraki, Japan) respectively, were amplified by PCR. AtPDK1 was cloned in the expression vector pGEX-4T-2 (Amersham, Little Chalfont, UK) and AtS6k2 in pMalC2X (New England Biolabs, Beverly, MA, USA). Substitutions of Asp-167, Thr-176, Ser-177, Thr-211, Ser267 and Ser-382 with Ala in AtPDK1 cDNA was accomplished by PCR-based mutagenesis using the QuickChange ${ }^{\text {TM }}$ procedure (Stratagene, Cedar Creek, TX, USA). All constructs were checked by DNA automated sequencing.

Recombinant glutathione S-transferase (GST) and maltose binding protein (MBP) fusion proteins were expressed in the Escherichia coli strain BL21(DE3). The E. coli cultures were grown at $30{ }^{\circ} \mathrm{C}, 220 \mathrm{rpm}$, until an $\mathrm{OD}_{600}$ of 0.6. GST-AtPDK1 was induced with $30 \mu \mathrm{M}$ isopropyl- $\beta$-D-1thiogalactoside (IPTG) at $16{ }^{\circ} \mathrm{C}$ over night and MBPAtS6k2 with $0.3 \mathrm{mM}$ IPTG at $30{ }^{\circ} \mathrm{C}$ for $5 \mathrm{~h}$. The GST and MBP fusion proteins were affinity-purified using glutathioneSepharose 4B or amylose resin, respectively, according to the manufacturer's instructions with $2.5 \mathrm{mM} 1,4$ dithiothreitol (DTT) and 0.1 $\mu \mathrm{M}$ PMSF added. GST fusion proteins (or GST control) were either kept bound to resin or eluted with $10 \mathrm{mM}$ glutathione, $50 \mathrm{mM}$ Tris- $\mathrm{HCl}, \mathrm{pH} 8.0$, or cleaved from the GST-tag with thrombin (Amersham, Little Chalfont, UK) at $20 \pm 2{ }^{\circ} \mathrm{C}$ for $2 \mathrm{~h}$ according to the manufacturer's instructions. MBP fusion proteins were eluted with $10 \mathrm{mM}$ maltose, $0.2 \mathrm{M} \mathrm{NaCl}, 1 \mathrm{mM}$ EDTA, $20 \mathrm{mM}$ Tris$\mathrm{HCl}, \mathrm{pH} 7.4$.

14-3-3 cDNAs were kindly provided by R. J. Ferl (Biotechnology and Horticultural Sciences, University of Florida, Gainesville, USA). Expression and purification of 12 14-33 isoforms was carried out according to the PET System manual (Novagen, Madison, WI, USA) as described previously [24]. Protein purification was carried out by immobilised metal ion affinity chromatography using a $\mathrm{Ni}^{2+}$-HiTrap chelating column (Pharmacia Biotech, Uppsala, Sweden), according to the manufacturer's protocol. The purified 14-33 protein buffer was exchanged using Microcon centrifugal filter devices (Millipore, Bedford, MA, USA) into $0.5 \mathrm{M}$ $\mathrm{NaCl}, 20 \mathrm{mM}$ Tris-HCl, pH 7.5, and 5\% (w/v) DMSO.

The protein concentration in the affinity isolates was determined according to Bearden [25] using BSA as a standard.

\subsection{Autophosphorylation of AtPDK1}

AtPDK1 or GST-AtPDK1 ( $3 \mu \mathrm{M}$, final concentration) was incubated in phosphorylation buffer $\left(2 \mathrm{mM} \mathrm{MgCl}_{2}, 0.2 \mathrm{mM}\right.$ 
$\mathrm{CaCl}_{2}, 2 \mu \mathrm{M}$ ATP, containing $\left[\gamma-{ }^{32} \mathrm{P}\right]$ ATP $(1700 \mathrm{dpm} / \mathrm{pmol}$ specific activity), $5 \mathrm{mM}$ Tris- $\mathrm{HCl}, \mathrm{pH} 7.5$ ) in a total volume of $14 \mu \mathrm{l}$. The reaction was carried out at $20 \pm 2{ }^{\circ} \mathrm{C}$ and terminated after $45 \mathrm{~min}$ with Laemmli sample buffer [26]. The polypeptides were separated by SDS-PAGE, using an $8 \%$ minigel, according to Laemmli [26] and stained with Coomassie Brilliant Blue R-250. A Phosphorimager (Amersham, Little Chalfont, UK) was used to visualise the $\left[\gamma-{ }^{32} \mathrm{P}\right]-$ polypeptides.

In time course experiments the phosphorylation reaction mixture was scaled up and samples withdrawn at indicated times.

\subsection{AtPDK1 phosphorylation of AtS6k2}

The phosphorylation assay mixture was as for autophosphorylation except that the potential substrate MBP-AtS6k2 (3 $\mu \mathrm{M}$ final concentration) was also included. As GSTAtPDK1 and MBP-AtS6k2 have approximately the same relative molecular masses, the MBP fusion tag was in some cases removed to improve the separation of the two proteins when run on SDS-PAGE. To remove the MBP fusion tag from AtS6k2, $1 \mu \mathrm{l}$ of factor Xa protease (New England Biolabs, Beverly, MA, USA) was added after 30 min phosphorylation followed by incubation for $2 \mathrm{~h}$ at $20 \pm 2{ }^{\circ} \mathrm{C}$ before terminating the reaction with Laemmli sample buffer [26]. SDSPAGE and further handling was as described above for autophosphorylation.

To test whether phospholipids influence the AtPDK1 phosphorylation of AtS6k2 $1 \mu$ l of phospholipid vesicles containing PtdCho $(100 \mu \mathrm{M}$, Sigma-Aldrich, Sweden $)$ and one of the phospholipids PtdSer, PtdOH $(100 \mu \mathrm{M}$ each, SigmaAldrich, Sweden), PtdIns3P, PtdIns5 $P, \operatorname{PtdIns}(3,4) P_{2}$, PtdIns $(3,5) P_{2}, \operatorname{PtdIns}(3,4,5) P_{3}(10 \mu \mathrm{M}$ each, Echelon, USA $)$, PtdIns $4 P$ or PtdIns $(4,5) P_{2}(10 \mu \mathrm{M}$ each, Sigma-Aldrich, Sweden) was added to the AtS6k2 phosphorylation assay described above.

To analyse the effect of 14-3-3 on AtPDK1 phosphorylation activity, His-tagged 14-3-3 proteins ( $3 \mu \mathrm{M}$, final concentration) were preincubated with GST-AtPDK1 for $2 \mathrm{~h}$ at $30^{\circ} \mathrm{C}$ in phosphorylation buffer followed by addition of MBPAtS6k2 and ATP to start the reaction. The samples were solubilised at room temperature in Laemmli sample buffer, and polypeptides were separated using SDS-PAGE, using a 10\% minigel, according to Laemmli [26]. The polypeptides were stained with Coomassie Brilliant Blue R-250 and a Phosphorimager (Amersham, Little Chalfont, UK) was used to visualise the $\left[\gamma-{ }^{32} \mathrm{P}\right]$-polypeptides. The phosphorylated bands were quantified using an image quant program (Amersham, Little Chalfont, UK).

\subsection{Phosphatase treatment of AtPDK1}

Resin-bound GST-AtPDK1 (15 $\mu \mathrm{M}$, final concentration) was incubated for $10 \mathrm{~min}$ in a $21 \mu \mathrm{l}$ autophosphorylation assay solution, as described above. The resin was then washed three times in $50 \mu \mathrm{l}$ (50 mM Tris- $\mathrm{HCl}, \mathrm{pH} 8.5,0.1 \mathrm{mM}$ EDTA) before addition of $1 \mu \mathrm{l}$ alkaline phosphatase (18 units per $\mu \mathrm{l}$, Roche Diagnostics, Germany). After $2 \mathrm{~h}$ treatment at $20^{\circ} \mathrm{C}$, the resin was washed five times in $50 \mu$ phosphorylation buffer without ATP. The dephosphorylated samples were again subjected to phosphorylation, by the addition of $2 \mu \mathrm{M}$ $\left[\gamma_{-}{ }^{32} \mathrm{P}\right]$ ATP $(1700 \mathrm{dpm} / \mathrm{pmol}$ specific activity) and the reaction terminated after $2 \mathrm{~h}$ with Laemmli sample buffer [26]. Samples collected before and after addition of phosphatase treatment, as well as after the final phosphorylation assay, were subjected to SDS-PAGE and analysed as described above.

\subsection{In situ proteolytic digestion}

GST-PDK1 was phosphorylated as described above, but without addition of radiolabelled ATP. The reaction mixture was separated by SDS-PAGE and a Coomassie Brilliant Bluestained protein band was excised from the gel at a molecular mass corresponding to the band detected by $\left[{ }^{32} \mathrm{P}\right]-$ phosphorylation. The gel band was cut into $1.5 \mathrm{~mm}$ pieces that were washed by incubation in $100 \mu \mathrm{l} 50 \%$ (v/v) ethanol,

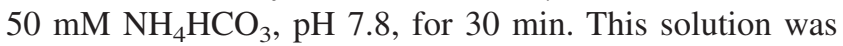
replaced by $75 \mu \mathrm{l}$ ethanol. After $10 \mathrm{~min}$ incubation the ethanol was removed and $10 \mu \mathrm{l} 10 \mathrm{mM}$ DTT, $50 \mathrm{mM} \mathrm{NH}_{4} \mathrm{HCO}_{3}$, $\mathrm{pH} 7.8$, were added for reduction of cysteine disulphides. The gel sample was incubated for $30 \mathrm{~min}$ at $37^{\circ} \mathrm{C}$ and then washed in $100 \%$ ethanol as described above. For carbamidomethylation of cysteine residues, $10 \mu \mathrm{l} 55 \mathrm{mM}$ iodoacetamide, $50 \mathrm{mM}$ $\mathrm{NH}_{4} \mathrm{HCO}_{3}, \mathrm{pH} 7.8$, were added and the sample was incubated for $30 \mathrm{~min}$ in the dark. Following $10 \mathrm{~min}$ incubation in $50 \%$ (v/v) ethanol, $50 \mathrm{mM} \mathrm{NH}_{4} \mathrm{HCO}_{3}, \mathrm{pH} 7.8$, and washing in ethanol as described above, the gel pieces were dried for $15 \mathrm{~min}$ in a vacuum centrifuge. The Eppendorf tubes containing the dried gel pieces were then put on ice and gel pieces were covered with $6 \mu \mathrm{l}$ of trypsin solution $(10 \mathrm{ng} / \mu \mathrm{l}$ recombinant trypsin from Pichia pastoris, Roche, Mannheim, Germany, in $50 \mathrm{mM} \mathrm{NH}_{4} \mathrm{HCO}_{3}$ ). Following a 30 min incubation step, during which the trypsin solution was absorbed by the gel particles, another $12 \mu \mathrm{l}$ of $50 \mathrm{mM} \mathrm{NH}_{4} \mathrm{HCO}_{3}$ was added and the samples were incubated over night at $37^{\circ} \mathrm{C}$. To stop proteolysis and assist peptide extraction, $18 \mu \mathrm{l} 0.5 \%$ (v/v) trifluoroacetic acid (TFA), and $2 \mathrm{mM}$ octyl- $\beta$-D-glucopyranoside was added. After 30 min incubation, the supernatant was aspired from the gel pieces and transferred to separate Eppendorf tubes.

\subsection{Derivatisation of phosphopeptides}

The protein digest sample was lyophilised in a vacuum centrifuge and resuspended in 30\% (v/v) 1-propanol, $20 \mathrm{mM}$ $\mathrm{Ba}(\mathrm{OH})_{2}, 0.5 \mathrm{M}$ heptanethiol for the one-reaction procedure of base-catalysed $\beta$-elimination of serine- and threoninephosphoryl groups, followed by addition of the heptanethiol moiety at the phosphorylation site [27]. The samples were incubated at $45^{\circ} \mathrm{C}$ for $3 \mathrm{~h}$ and the reaction was stopped by 
acidifying each sample with $0.5 \mu \mathrm{l} 10 \%(\mathrm{v} / \mathrm{v})$ TFA and by precipitation of $\mathrm{Ba}^{2+}$-ions with $\mathrm{NH}_{4}\left(\mathrm{SO}_{4}\right)$ at 10 -fold molar excess. Samples were then centrifuged at $15700 \times g$ for $5 \mathrm{~min}$ and supernatants were collected and lyophilised over night in a vacuum centrifuge. The derivatised samples were analysed by MALDI-TOF MS in parallel with non-derivatised samples.

\subsection{MALDI-TOF mass spectrometry}

Peptide extracts of derivatised and non-derivatised tryptic peptides were analysed by matrix-assisted laser desorption/ ionisation mass spectrometry (MALDI-TOF MS) and selected peptides were analysed by MALDI post-source decay or collision-induced dissociation fragment ion analysis for elucidation of individual phosphorylation sites. The lyophilised samples were each resuspended in $10 \mu \mathrm{l} 0.1 \%$ (v/v) TFA of which $1 \mu \mathrm{l}$ was prepared on the MALDI sample support using $\alpha$-4-hydroxycinnamic acid as the MALDI matrix. Mass spectrometric measurements were performed either on a Bruker Ultraflex MALDI TOF/TOF instrument (Bruker Daltonics, Germany) or on a 4700 proteomics analyser MALDI TOF/ TOF instrument (Applied Biosystems, Framingham, MA, USA). On average, the presented spectra from the Bruker Ultraflex are the sums of 500 and 2000 single-shot spectra for MS and MS/MS mode, respectively. The presented MS/MS-spectrum in Fig. 3C, measured on the 4700 proteomics analyser, is the sum of 55000 single-shot spectra. Mass spectra of positively charged ions were acquired in the reflector mode using delayed ion extraction. The calibration constants were determined using internal standard peptides included in the matrix solution, resulting in an error of $<50 \mathrm{ppm}$ for the recorded mass spectra. The obtained peptide mass fingerprint data and fragment ion mass data were evaluated by searching the NCBInr database using the software package Mascot (Matrix Science, UK), allowing for the variable modification of heptanethiol addition at serine and threonine residues $(+114.087 \mathrm{Da})$. The mass error tolerance for fragment ion masses was set to $0.8 \mathrm{Da}$ for data from the Bruker Ultraflex and 0.2 Da for data from the Applied Biosystems 4700 proteomics analyser. The fragment ion mass data were evaluated manually for elucidation of phosphorylation sites.

\subsection{Protein sequence analysis}

Alignment of amino acid sequences was performed using the Clustal X program available from the ExPasy Molecular Biology Server (www.expasy.ch). The protein sequences can be accessed in the NCBI database (www.ncbi.nlm.nih.gov/). The NCBI accession numbers are for A. thaliana PDK1 AF132742, Oryza sativa PDK1 AF132743, mouse PDK1 Q9Z2AO, and human PDK1 O15530.

\section{Results}

The aim of this study was to identify potential AtPDK1 substrates and to characterise the AtPDK1 enzyme with respect to the importance of autophosphorylation for its activity. Identification of autophosphorylation sites as well as of other sites and their respective importance for autophosphorylation of AtPDK1 and further downstream phosphorylating capacities was also addressed. As a first step we tested whether AtPDK1 is capable of autophosphorylation and of phosphorylation of its proposed substrate AtS6k2.

\subsection{AtPDK1 autophosphorylation and phosphorylation of AtS6k2}

E. coli-expressed GST-tagged AtPDK1 was subjected to phosphorylation conditions, and analysed by autoradiography. GST-AtPDK1 was phosphorylated in the presence of $\left[\gamma_{-}{ }^{32} \mathrm{P}\right]$ ATP (Fig. 1A), indicating that it is autophosphorylated. This phosphorylation increased linearly with time (Fig. 1A).

To verify that the phosphorylation of AtPDK1 represents autophosphorylation, the phosphorylated enzyme was subjected to alkaline phosphatase treatment (Fig. 1B, lane 2). After dephosphorylation, resin-bound GST-AtPDK1 was again subjected to phosphorylation by addition of $\left[\gamma_{-}{ }^{32} \mathrm{P}\right]$ ATP. As seen in Fig. 1B, lane 3, GST-AtPDK1 exhibited a strong phosphorylating activity after phosphatase treatment. At-

\section{A}

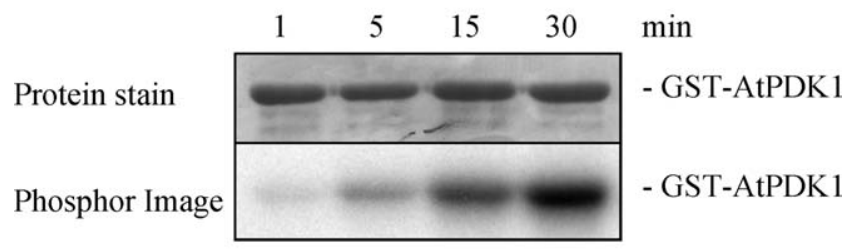

B

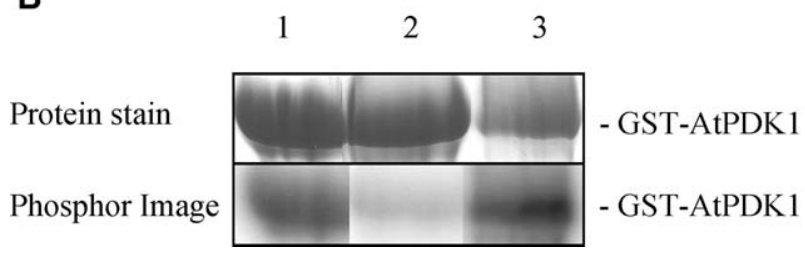

Fig. 1. Autophosphorylation of AtPDK1.

(A) Time-dependent phosphorylation of AtPDK1.

GST-AtPDK1 was incubated in the presence of $\left[\gamma_{-}{ }^{32} \mathrm{P}\right]$ ATP. Reactions were terminated after 1-30 min with Laemmli sample buffer and individual samples separated by SDS-PAGE. The polypeptides were stained with Coomassie Brilliant Blue R-250 (upper panel) and a Phosphorimager was used to visualise the ${ }^{32} \mathrm{P}$-polypeptides (lower panel). Similar results were obtained in three separate experiments.

(B) AtPDK1 is autophosphorylated after dephosphorylation by alkaline phosphatase.

Resin-bound GST-AtPDK1 was incubated in the presence of $\left[\gamma_{-}{ }^{32} \mathrm{P}\right]$ ATP. After $10 \mathrm{~min}$, the resin was washed and alkaline phosphatase was added. After $2 \mathrm{~h}$ treatment the resin was washed and $\left[\gamma_{-}{ }^{32} \mathrm{P}\right]$ ATP added. The reaction was terminated after $2 \mathrm{~h}$ by addition of Laemmli sample buffer and individual samples separated by SDS-PAGE. The polypeptides were stained with Coomassie Brilliant Blue R-250 (upper panel) and a Phosphorimager was used to visualise the ${ }^{32} \mathrm{P}$-polypeptides (lower panel). Samples were taken out before (lane 1) and after (lane 2) alkaline phosphatase treatment as well as after the final phosphorylation assay (lane 3). Similar results were obtained in two separate experiments. 
PDK1 was also phosphorylated when cleaved from the GSTtag (Fig. 2, lane 1). Taken together, these results verify that the phosphorylation observed is due to an intrinsic kinase activity of AtPDK1 and excludes involvement of protein kinases, unspecifically bound to GST during preparation.

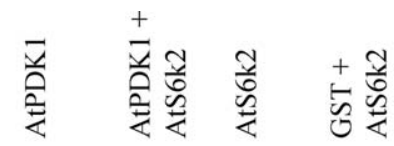

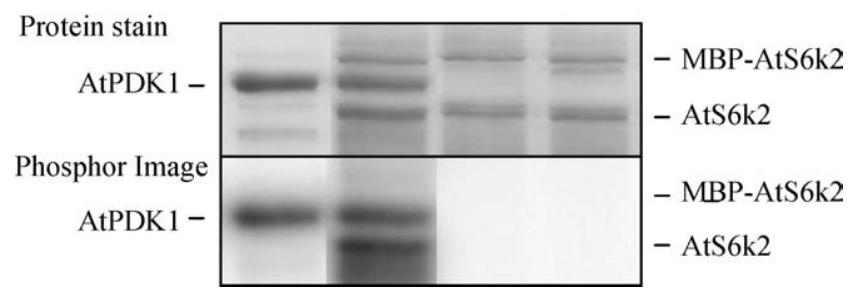

Fig. 2. Autophosphorylated AtPDK1 catalyses phosphorylation of AtS6k2. AtPDK1 and MBP-AtS6k2 were incubated in the presence of $\left[\gamma_{-}{ }^{32} \mathrm{P}\right]$ ATP. After $30 \mathrm{~min}$ the MBP-tag was removed from AtS6k2. Reactions were terminated after $2.5 \mathrm{~h}$ with Laemmli sample buffer and individual samples separated by SDS-PAGE. The polypeptides were stained with Coomassie Brilliant Blue R-250 (upper panel) and a Phosphorimager was used to visualise the ${ }^{32}$ P-polypeptides (lower panel). Lane 1: AtPDK1, Lane 2: AtPDK1 and AtS6k2, Lane 3: AtS6k2, Lane 4: GST and AtS6k2. Similar results were obtained in four separate experiments. Note; the GST band is not shown since it migrates at $\sim 26 \mathrm{kDa}$. AtPDK1 and AtS6k2 predicted molecular masses are $54.7 \mathrm{kDa}$ and $53.1 \mathrm{kDa}$, respectively.
No phosphorylation of AtS6k2 was observed in the presence of $\left[\gamma-{ }^{32}\right.$ P]ATP (Fig. 2, lane 3). However, upon addition of AtPDK1 to the mixture, a phosphorylation of AtS6k2 was obtained (Fig. 2, lane 2). The AtPDK1-induced phosphorylation of AtS6k2 was clearly detectable already after $1 \mathrm{~min}$ (data not shown) and increased with time. These results clearly indicate that AtS6k2 is a potential substrate for AtPDK1.

\subsection{Identification of phosphorylation sites in AtPDK1 by mass spectrometry}

PDK1s are commonly phosphorylated at several sites, some of which are important for enzyme activity, others for substrate association as well as for binding of regulatory molecules such as 14-3-3 proteins [14-16,22]. We aimed at identifying phosphorylation sites in AtPDK1 and their participation in either its activity, or in the interaction and/or phosphorylation of AtS6k2. E. coli-expressed GSTAtPDK1 was phosphorylated with non-radiolabelled ATP followed by separation of the mixture by SDS-PAGE after which the GST-AtPDK1 band was cut out and trypsinised. To determine serine and threonine phosphorylation sites in AtPDK1, we employed a technique based on $\beta$-elimination of the peptide phosphoryl groups followed by addition of heptanethiol to the so formed dehydroalanyl (from phosphoserine) or dehydroamino-2-butyric acid (from phosphothreonine) residues [27]. The combined elimination of a phosphoryl group

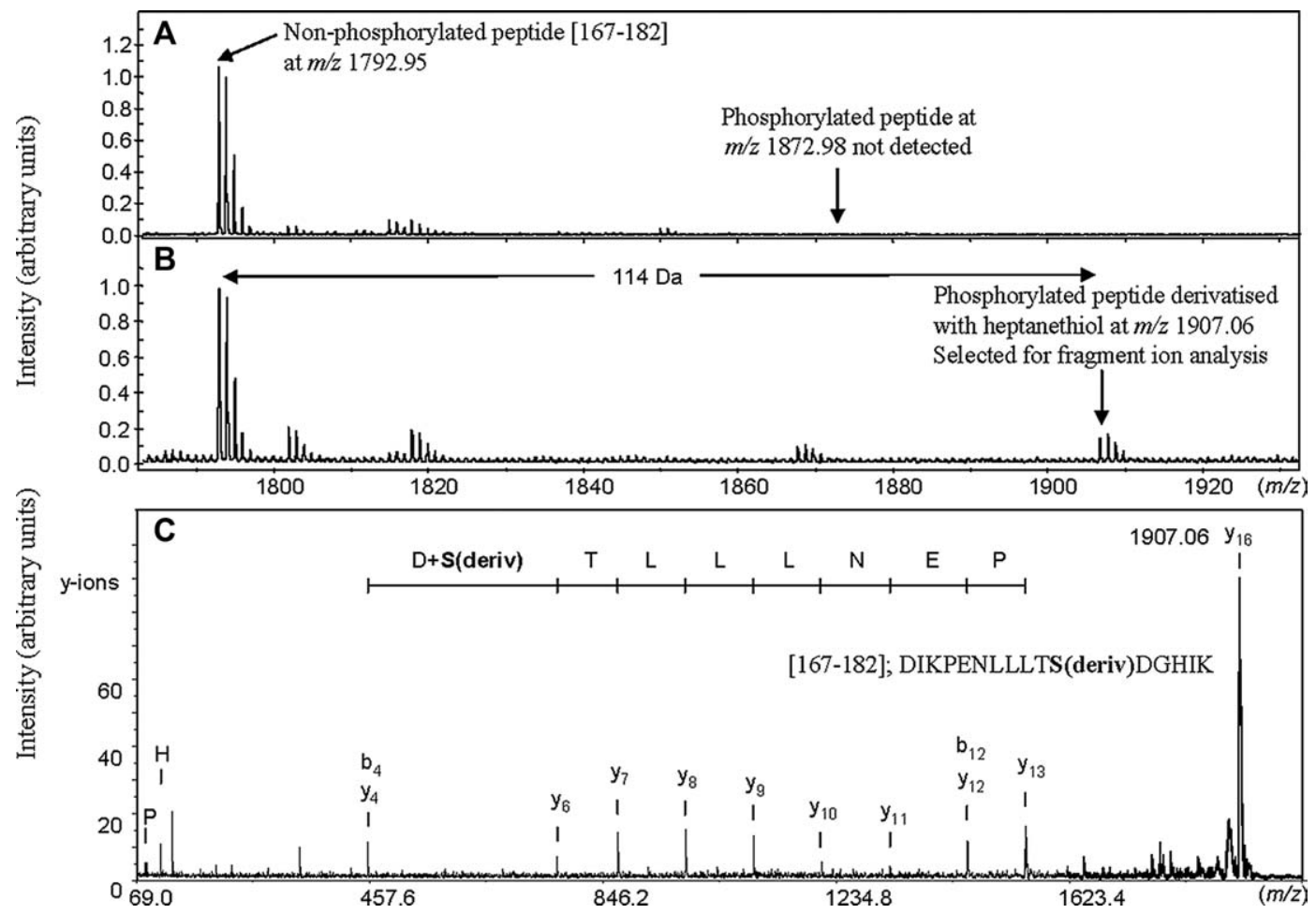

Fig. 3. Identification of Ser-177 as an AtPDK1 autophosphorylation site by MALDI MS analysis.

The top panels show a selected region of the mass spectra corresponding to (A) the underivatised peptide mixture, and (B) the peptide mixture after derivatisation of phosphopeptides by heptanethiol. The signal at $\mathrm{m} / \mathrm{z} 1792.95$ in both spectra matches the calculated molecular mass of the unphosphorylated tryptic peptide 167-182 (DIKPENLLLTSDGHIK) of AtPDK1. In B, and additional signal at $\mathrm{m} / \mathrm{z} 1907.06$ was detected, matching the calculated molecular mass of the same peptide derivatised by heptanethiol, thus indicating it as being phosphorylated in the original sample. This peptide was subjected to fragment ion analysis (C). The detected y-ion series, indicated in the figure, confirmed the identity of the peptide and located the phosphorylation site to Ser-177. 

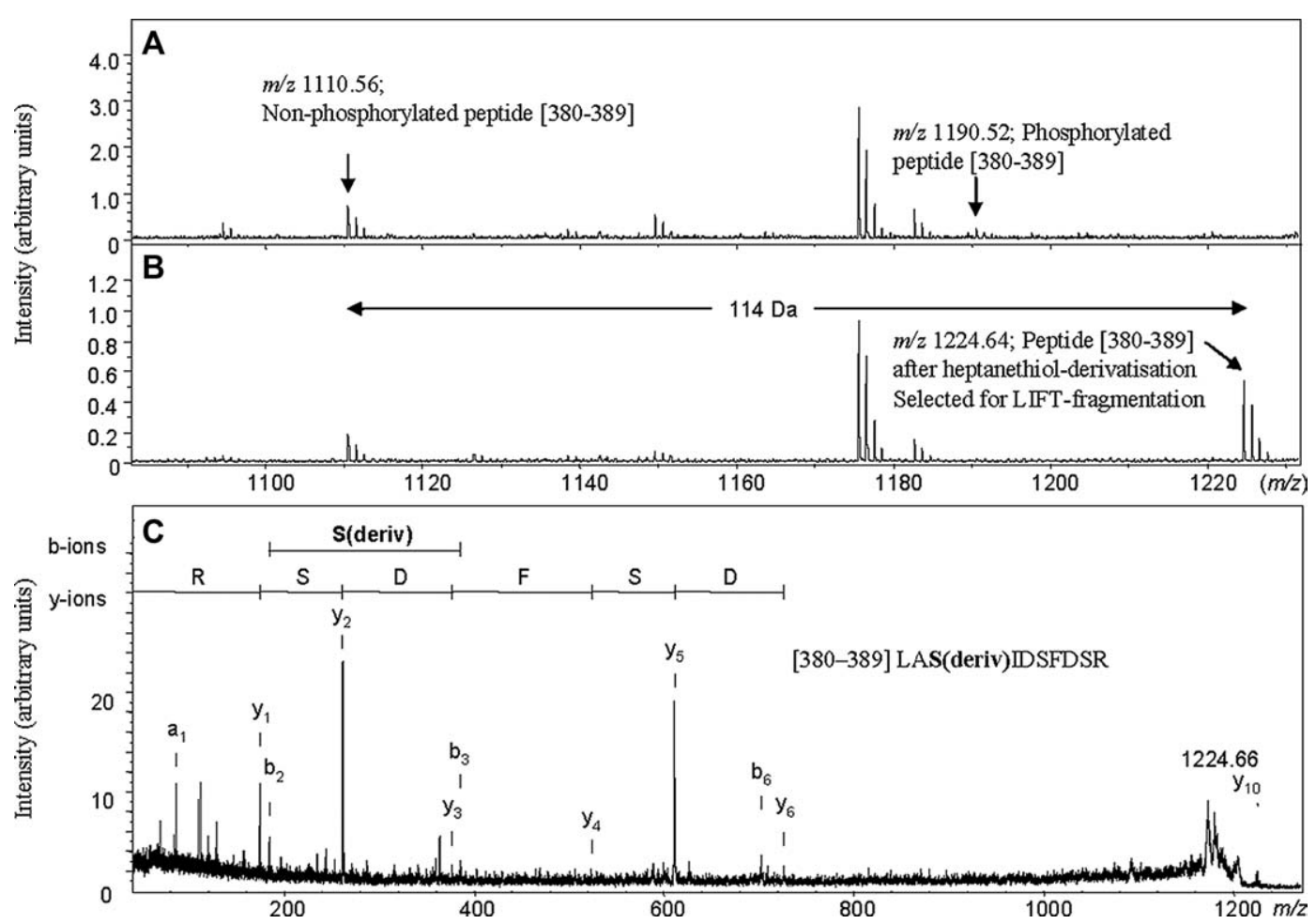

Fig. 4. Identification of Ser-382 as an AtPDK1 autophosphorylation site by MALDI MS analysis.

The top panels show a selected region of the mass spectra corresponding to (A) the underivatised peptide mixture, and (B) the peptide mixture after derivatisation of phosphopeptides by heptanethiol. The signal at $\mathrm{m} / z, 1224.64$ in B, matches the calculated molecular mass of the phosphorylated AtPDK1 tryptic peptide 380-389 (LASIDSFDSR) derivatised with heptanethiol. This peptide was subjected to fragment ion analysis (C). The detected b- and y- ion series, indicated in the figure, confirmed the identity of the peptide and located the phosphorylation site to Ser-382.

and the addition of a heptanethiol moiety result in a net increase of the peptide molecular mass by 34.12 Da compared to the original phosphorylated peptide. Compared to its non-phosphorylated counterpart, a phosphorylated peptide derivatised with heptanethiol thus increases in mass by 114.09 Da. This derivatisation technique has the advantage over direct analysis of phosphorylated peptides that the negatively charged phosphoryl group, which gives the peptide low ionisation efficiency in MALDI, is exchanged for a neutral aliphatic heptanethiol moiety. This improves the detection sensitivity for phosphorylated peptides by MALDI MS. Indeed, using this technique, we were able to detect a phosphorylated peptide in the heptanethiol-derivatised phosphorylated AtPDK1 sample, which was not detected in the nonderivatised sample (Fig. 3A and B). This tryptic peptide; AspIle-Lys-Pro-Glu-Asn-Leu-Leu-Leu-Thr-Ser-Asp-Gly-His-IleLys (DIKPENLLLTSDGHIK), corresponding to amino acids 167-182, contains one threonine and one serine residue, both potential phosphorylation sites. To elucidate which of these two sites was phosphorylated, the derivatised peptide was subjected to fragment ion mass analysis (Fig. 3C). The extensive series of $y$-ions indicated in the top of the panel (Fig. 3C), encompasses both the $\mathrm{y}_{6}$ - and $\mathrm{y}_{7}$-ions, which contain the derivatised Ser-177 and the non-derivatised Thr-176 residues, respectively. No signal corresponding to the $\mathrm{y}_{6}$-ion of the nonphosphorylated form of Ser-177 was detected.

In another tryptic peptide, Leu-Ala-Ser-Ile-Asp-Ser-PheAsp-Ser-Arg (LASIDSFDSR, amino acids 380-389) obtained from the phosphorylated AtPDK1, we could identify the phosphorylation site among the three possible serine residues (Fig. 4). In this case, the $b_{3}$-ion, which contains the derivatised serine residue, together with the y-ion series, which cover both the two non-derivatised serine residues Ser-285 and Ser288, clearly indicated phosphorylation at Ser-382. Internal fragment ions covering the Ser-382 supported this conclusion (data not shown). A third phosphorylation site was deduced from the 114.09 Da mass increment for the peptide Asp-Ile-Lys-Phe-Pro-Asn-His-Phe-Ser-Glu-Ala-Ala-Arg (DIKFPNHFSQAAR, amino acids 268-280), which contains only one possible phosphorylation site, Ser-276 (data not shown). No signals corresponding to peptides with multiple additions of heptanethiol were detected in the MALDITOF MS analyses.

The three identified autophosphorylation sites, Ser-177, Ser-276 and Ser-382, were also identified without the addition of ATP to the phosphorylation assay, which shows that the expressed AtPDK1 is able to autophosphorylate in E. coli.

By comparing the amino acid sequence of PDK1 from several different organisms, it can be seen that all three autophosphorylation sites identified by mass spectrometry are conserved between the plant isoforms in rice and A. thaliana (Fig. 5). In contrast, the amino acid residues in mammalian PDK1 isoforms corresponding to Ser-177 and Ser-276 in AtPDK1, can not be phosphorylated (glutamic acid and phenylalanine, respectively) (Fig. 5). 


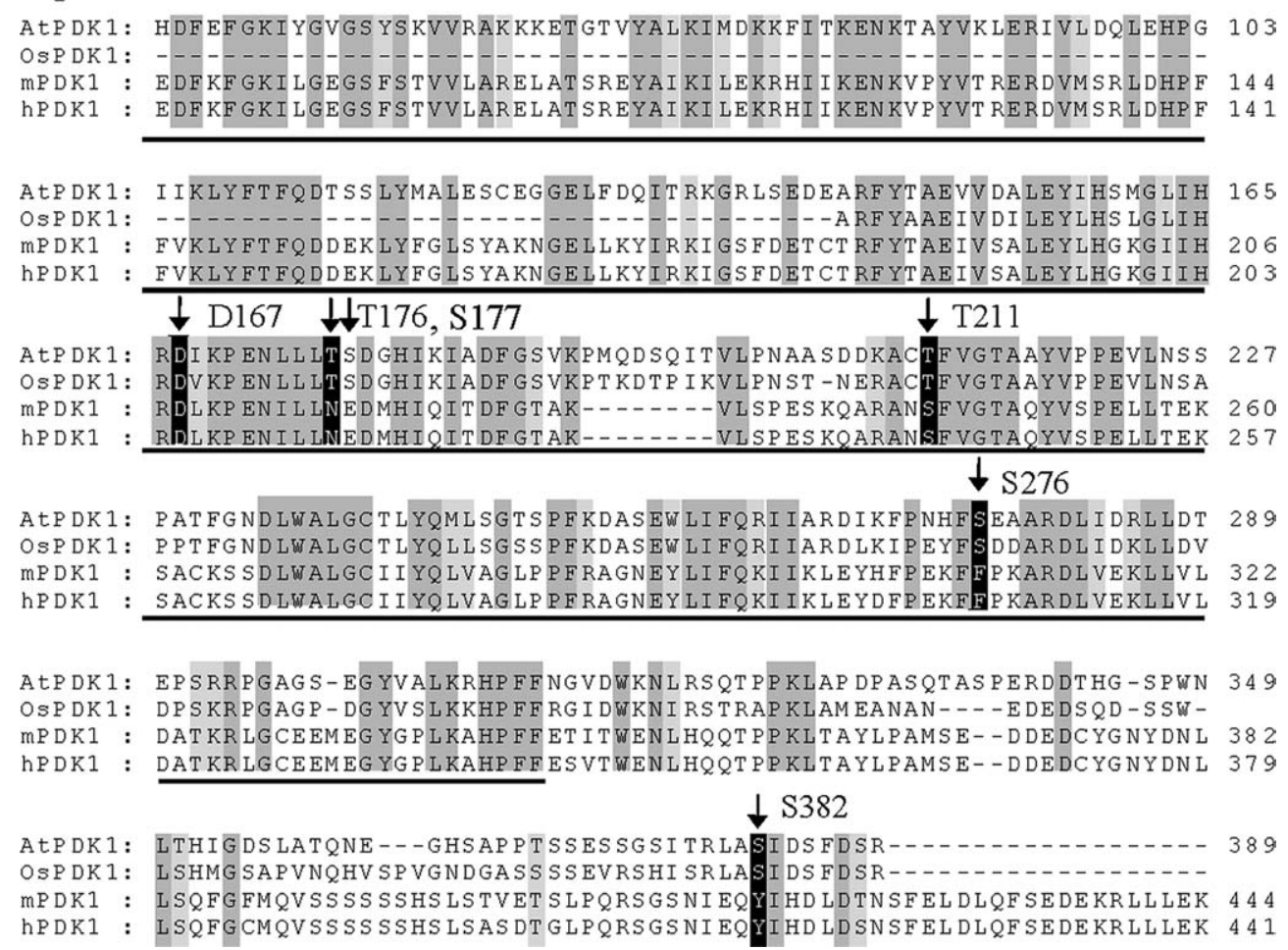

Fig. 5. Alignment of the amino acid sequences of PDK1 isoforms.

The figure shows alignment with amino acid sequences corresponding to residues 80-449 in human PDK1 (hPDK1). The NCBI accession numbers are for $A$. thaliana PDK1 (AtPDK1) AF132742, rice PDK1 (OsPDK1) AF132743, mouse PDK1 (mPDK1) Q9Z2AO and hPDK1 AF017995. The alignment was carried out using the Clustal X program. Identities are shaded in dark grey and similarities in light grey. The underlined residues correspond to the catalytic domain. Residues investigated in this work are indicated with arrows. No numbering is given for the rice PDK1 sequence as the full-length sequence is not known.

\subsection{The role of the three identified and two potential AtPDK1 phosphorylation sites}

To further characterise the role of AtPDK1 autophosphorylation sites identified by mass spectrometry, we used sitedirected mutagenesis to exchange these residues for alanines. Interestingly, the Ser276Ala mutant was not autophosphorylated (Fig. 6A). In addition, this mutant was not able to phosphorylate AtS6k2 (Fig. 6B). These results indicate that phosphorylation of Ser-276 in AtPDK1 is essential for downstream phosphorylating activity. The Ser177Ala autophosphorylation and phosphorylation of AtS6k2 was drastically lowered compared to wild-type AtPDK1 (Fig. 6). The Ser382Ala mutant was autophosphorylated to a somewhat lower degree but phosphorylated AtS6k2 to a similar level as the wild-type (Fig. 6).

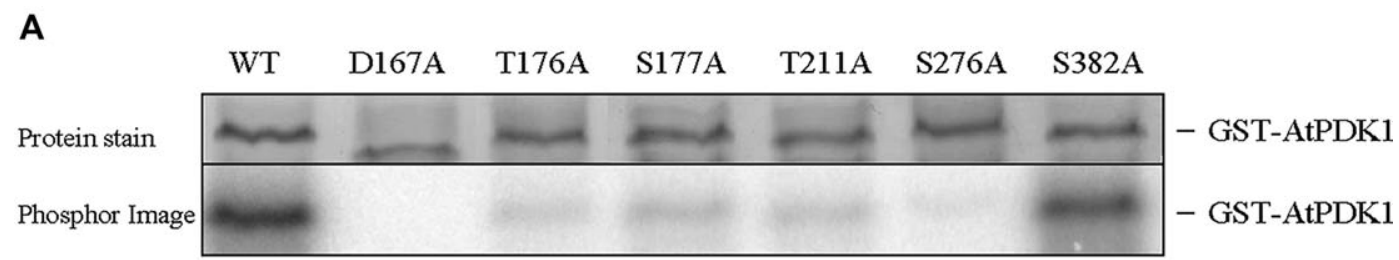

B

\begin{tabular}{|c|c|c|c|c|c|c|c|c|}
\hline & WT & D167A & T176A & S177A & $\mathrm{T} 211 \mathrm{~A}$ & S276A & S382A & \\
\hline Protein stain & E. & $z$ & & $4+4$ & 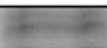 & & 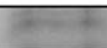 & - AtS6k2 \\
\hline 1osphor Image & & & & & & & & - \\
\hline
\end{tabular}

Fig. 6. Effect of AtPDK1 mutations on autophosphorylation and AtS6k2 phosphorylation.

GST-bound AtPDK1 mutant proteins were assayed for autophosphorylation in the presence of $\left[\gamma-{ }^{32} \mathrm{P}\right] \mathrm{ATP}(\mathbf{A})$ ). To examine the effect on AtS6k2 phosphorylation, MBP-AtS6k2 was added to the reactions in (B). After 30 min the MBP-tag was removed from AtS6k2. Reactions were terminated after 2.5 h with Laemmli sample buffer and individual samples separated by SDS-PAGE. The polypeptides were stained with Coomassie Brilliant Blue R-250 (upper panels) and a Phosphorimager was used to visualise the ${ }^{32} \mathrm{P}$-polypeptides (bottom panels). Similar results were obtained in four separate experiments. 
In human PDK1, autophosphorylation of Ser-241 is critical for kinase activity [13]. The corresponding residue in AtPDK1 is Thr-211 (Fig. 5). However, in this study, no peptide containing Thr-211 (neither unmodified nor modified) in AtPDK1 was detected by mass spectrometry. To determine whether Thr-211 represents an important site also in AtPDK1, a Thr211Ala mutant was constructed. As seen in Fig. 6, the Thr211Ala mutant was seriously affected in its capability to autophosphorylate itself and to phosphorylate AtS6k2 compared to both wild-type AtPDK1 and the Ser382Ala mutant.

Another potential phosphorylation site could be the Thr176 located next to the identified Ser-177. Phosphorylation of Thr-176 was not detected by mass spectrometry. However, the Thr176Ala mutant seriously affected AtPDK1 autophosphorylation and phosphorylation of AtS6k2 indicating its importance for activity (Fig. 6). Unfortunately, we could not identify the phosphorylated amino acids present in the Thr176Ala, Thr211Ala or Ser382Ala mutants.

\subsection{Characterisation of Asp-167 as an essential site for AtPDK1 activity}

The catalytic domain of PDK1 is highly conserved among species (Fig. 5). One of the conserved residues, Asp-205 in human PDK1, has been suggested to be important for activity (annotation in NCBI database, www.ncbi.nlm.nih.gov/, accession number O15530). This residue is situated in a DL/IKPEN motif (Fig. 5) and has been shown essential for activity in all protein kinases tested. We used site-directed mutagenesis to exchange also this residue in AtPDK1 for an alanine. The resulting Asp167Ala mutant exhibited no autophosphorylating activity and no phosphorylation of the substrate AtS6k2 was detected (Fig. 6), confirming that this residue is essential for AtPDK1 activity and blocks autophosphorylation of the enzyme.

\subsection{Regulation of AtPDK1 phosphorylation by 14-3-3 isoforms}

Because mammalian PDK1 has been demonstrated to bind the two human 14-3-3 isoforms $\theta$ and $\eta$ [22], and to be negatively regulated by 14-3-3 $\theta$, we investigated the influence of 12 different $A$. thaliana 14-3-3 isoforms on AtPDK1 phosphorylation activity. Several of the 14-3-3 isoforms had a significant effect on the AtPDK1 autophosphorylation (Fig. 7). Nine of the 12 isoforms ( $\mu, \phi, \kappa, \omega, \epsilon, \psi, v, \chi$, and $v$ ) enhanced AtPDK1 autophosphorylation by $10-40 \%$, depending on isoform. This activation also enhanced AtS6k2 phosphorylation (Fig. 7). Two isoforms, $\lambda$ and 1 , had no apparent effect on AtPDK1 phosphorylation activity. Interestingly, one 14-33 isoform, o, markedly suppressed the AtPDK1 phosphorylation and consequently also the AtS6k2 phosphorylation to a barely detectable level.

\subsection{Effect of phospholipids on AtPDK1 autophosphorylation and phosphorylation of AtS6k2}

As AtPDK1 binds to several phospholipids in vitro [4], we tested the phospholipid dependency of AtPDK1 autophosphorylation and phosphorylation of AtS6k2. As seen in Fig. 8,

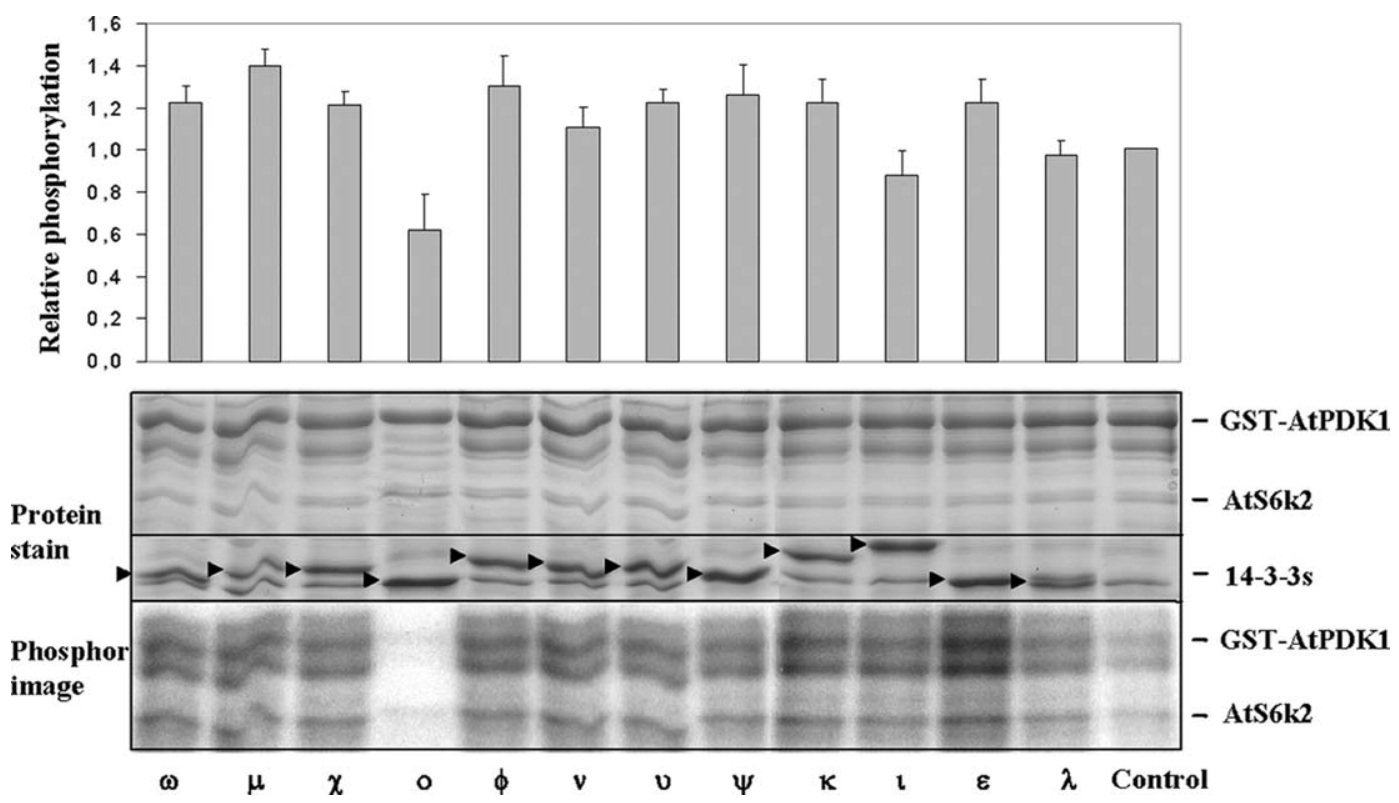

Fig. 7. Effect of 14-3-3 isoforms on AtPDK1 autophosphorylation and AtS6k2 phosphorylation.

GST-bound AtPDK1 was assayed for autophosphorylation and phosphorylation of AtS6k2 in the presence of $\left[\gamma_{-}{ }^{32} \mathrm{P}\right] \mathrm{ATP}$ and His-tagged $14-3-3$ isoforms. After 30 min the MBP-tag was removed from AtS6k2. Reactions were terminated after $2.5 \mathrm{~h}$ with Laemmli sample buffer and individual samples separated by SDS-PAGE. The polypeptides were stained with Coomassie Brilliant Blue R-250 (middle panels). 14-3-3 isoforms (28-31 kDa) are indicated with arrowheads. A Phosphorimager was used to visualise the ${ }^{32} \mathrm{P}$-polypeptides (lower panel). The phosphorylated bands were quantified using an Image Quant program and the GST-ATPDK1 band intensities were compared to the control, given a value of 1.0. The diagram (upper panel) shows mean values from five separate experiments. 


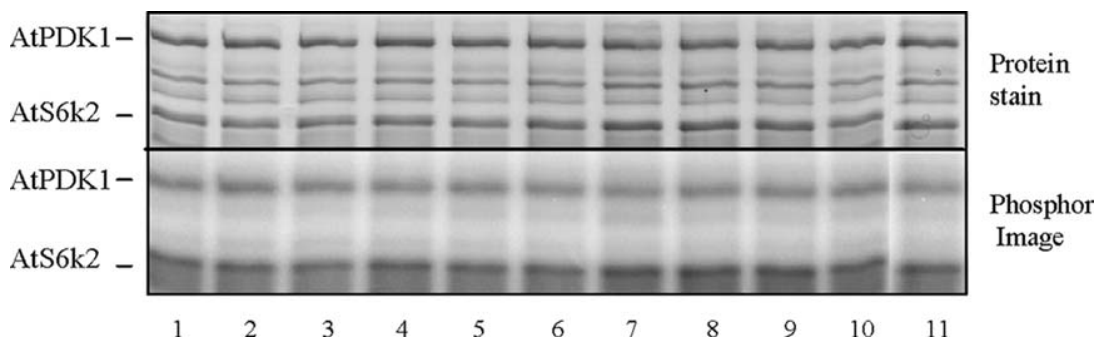

Fig. 8. Phospholipid effects on AtPDK1 phosphorylation of AtS6k2.

GST-AtPDK1 and MBP-AtS6k2 were incubated in the presence of $\left[\gamma_{-}{ }^{32} \mathrm{P}\right] \mathrm{ATP}$ and lipid vesicles of PtdCho and an additional phospholipid. After 30 min the MBP-tag was removed from AtS6k2. Reactions were terminated after $2.5 \mathrm{~h}$ with Laemmli sample buffer and individual samples separated by SDS-PAGE. The polypeptides were stained with Coomassie Brilliant Blue R-250 (upper panel) and a Phosphorimager was used to visualise the ${ }^{32} \mathrm{P}-$ polypeptides (lower panel). Lane 1: control (buffer), Lane 2: PtdIns3 $P$, Lane 3: PtdIns4P, Lane 4: PtdIns5 $P$, Lane 5: PtdIns(3,4) $P_{2}$, lane 6: PtdIns(3,5) $P_{2}$, Lane 7: PtdIns(4,5) $P_{2}$, Lane 8: PtdIns(3,4,5) $P_{3}$, Lane 9: PtdOH, Lane 10: PtdSer, Lane 11: PtdCho control. Similar results were obtained in three separate experiments.

none of the phospholipids tested (including phosphoinositides) significantly altered the phosphorylation levels of the two enzymes.

\section{Discussion}

In contrast to the mammalian PDK1, our knowledge of the plant PDK1 enzyme and its substrates is very limited. In this study we identify the A. thaliana S6 kinase AtS6k2 as a potential substrate for AtPDK1. As AtS6k2 can phosphorylate an A. thaliana ribosomal S6 protein [8], our findings suggest that the PDK1-S6K pathway, which regulates S6 and cell growth in animal systems [2], is also present in $A$. thaliana. Interestingly, in preliminary studies, we could detect a protein kinase able to phosphorylate AtS6k2 in an $A$. thaliana ribosomal preparation. By using an in-gel phosphorylation assay, where MBP-AtS6k2 was included in the gel, the molecular mass of this unknown protein kinase, was estimated to be $55 \mathrm{kDa}$ (data not shown), which correlates well with the predicted molecular mass of AtPDK1 (54.7 kDa).

An autophosphorylation of AtPDK1 was demonstrated in in vitro assays using $\left[\gamma_{-}{ }^{32} \mathrm{P}\right] \mathrm{ATP}$ (Fig. 1B). Furthermore, we showed that AtPDK1 becomes autophosphorylated and is constitutively active when expressed in E. coli, a feature in common with the mammalian PDK1 [13]. Three autophosphorylation sites were identified using MALDI-TOF MS, Ser-177, Ser-276 and Ser-382 (Figs. 3 and 4), and their functional importance was investigated by mutational analysis. Ser276 was crucial for AtPDK1 autophosphorylation and phosphorylation of AtS6k2, while mutation of Ser-177 did not completely abolish phosphorylation (Fig. 6). These two amino acids, located in the catalytic domain, are conserved in rice PDK1 but not in the mammalian PDK1 isoforms (Fig. 5). Interestingly, in mammalian PDK1, Ser-177 corresponds to a glutamic acid (Fig. 5), a residue that may mimic a phosphate group. The AtPDK1 mutant, Ser382Ala, was slightly less autophosphorylated, but phosphorylated AtS6k2 to a similar degree as the wild-type AtPDK1. Thus, this phosphorylation site, located outside the catalytic domain of AtPDK1, is not involved in AtS6k2 phosphorylation per se. However, other functions such as interactions with substrate, regulatory proteins or lipids cannot be ruled out at this stage.
All AGC kinases contain a T-loop, which must be phosphorylated to render the enzyme active [1]. In human PDK1, autophosphorylation of Ser-241, which resides in the T-loop, has been shown to be essential for activity [13]. Thr-211 in AtPDK1 corresponds to Ser-241 (Fig. 5). Substitution of Thr211 in AtPDK1 into alanine significantly reduced autophosphorylation of the enzyme and its phosphorylation activity towards AtS6k2 (Fig. 6). Interestingly, in mouse PDK1, substitution of Ser-244, corresponding to Ser-241 in human PDK1, with a threonine resulted in an overall increase in autophosphorylation [14]. The reduction in AtPDK1 autophosphorylation indicates the importance of Thr-211 for activity, although not as crucial as Ser-276. Whether Thr-211 represents a phosphorylation site or not remains to be clarified. The drastic reduction of autophosphorylation of the Thr211Ala mutant could be explained by this residue instead having a structural function. Another explanation could be that Thr-211 is not phosphorylated under the in vitro conditions used, in line with the observation with mouse PDK1, where the corresponding Ser-241 was phosphorylated to a higher degree under in vivo compared to in vitro conditions [14].

Taken together, identification of the Ser-276 as an autophosphorylation site essential for AtPDK1 activity, with corresponding phosphorylation site not present in mammalian PDK1, and with Thr-211 as a residue that may be less essential for activity than corresponding site in human PDK1 (phosphorylated), indicates that PDK1 enzymatic activity is likely to be differently regulated in plants and animals.

The more or less complete loss of autophosphorylation in the Ser177Ala and Ser276Ala mutant proteins, accompanied by a corresponding loss of AtS6k2 phosphorylation (Fig. 6), suggests that the $A$. thaliana PDK1, like its mammalian homologues, needs to be autophosphorylated to be able to affect downstream targets $[13,28]$. The mutational analysis of the three identified phosphorylation sites Ser-177, Ser-276 and Ser-382 also indicates that more than one site needs to be phosphorylated for full activity. The loss of autophosphorylation in Asp167Ala, Thr176Ala and Thr211Ala mutant proteins suggests that these residues are of importance for AtPDK1 activity and affect the autophosphorylation state of the enzyme. 
Recently, human 14-3-3 $\theta$ was shown to interact with PDK1 and reduced the activity via binding to Ser-241, a site corresponding to Thr-211 in AtPDK1 [22]. Thus, in the search for AtPDK1 interaction partners we tested the effect on its autophosphorylation by incubating AtPDK1 with 12 of the fifteen different Arabidopsis 14-3-3 isoforms identified [29]. For nine of the 12 isoforms tested, phosphorylation of AtPDK1 was enhanced, whereas one isoform, 14-3-3 o, significantly suppressed phosphorylation (Fig. 7). All 14-3-3s used in this study were shown to bind equally well in vitro to plasma membrane $\mathrm{H}^{+}$-ATPases, the major 14-3-3 target in the $A$. thaliana plasma membrane [30]. This suggests that the recombinant $14-3-3$ s are correctly folded and should be able to bind and affect other targets as well, e.g. PDK1. To resolve whether the differential effects on the AtPDK1 activity caused by the different 14-3-3 isoforms reflect isoform specificity further studies are required. Although all the 14-3-3 isoforms in A. thaliana show high sequence similarities, phylogenetic trees comparing amino acid sequences of 14-3-3 isoforms in A. thaliana as well as in other plants show that o is branched out from the other isoforms at an early stage [23], which may agree with this isoform having a distinct function. Most 14-33 isoforms are ubiquitously expressed in plants and 14-3-3 o is found in both roots and shoots in A. thaliana, but is less abundant compared to the other 14-3-3 isoforms [29]. Attempts to identify a 14-3-3 binding site in the AtPDK1 enzyme have so far been unsuccessful. We detect 14-3-3 binding with all isoforms but see no distinct difference between the GST control, the various 14-3-3 isoforms or the Thr211Ala mutant (mutation corresponding to the 14-3-3 binding site in human PDK1 [22]). At present we can not, however, rule out unspecific binding. Further studies including in vivo assays and identification of an AtPDK1 14-3-3 binding site will assist in the elucidation of the physiological role of AtPDK1 and 14-3-3 interaction.

The PDK1 interaction with its substrates and regulatory components is still poorly understood. However, both mammalian and A. thaliana PDK1 contain a PH domain, often involved in mediating protein-lipid interactions, and mammalian PDK1 activity towards certain downstream targets is greatly enhanced in the presence of $\operatorname{PtdIns}(3,4) P_{2}$ or PtdIns $(3,4,5) P_{3}[1]$. PtdIns $(3,4,5) P_{3}$ was shown to activate the AtPDK1 phosphorylation of a human PKB enzyme [4], although the significance of this finding is unclear since to date neither PKB nor PtdIns $(3,4,5) P_{3}$ have been identified in higher plant cells. However, AtPDK1 has been shown to bind to a number of phospholipids including $\operatorname{PtdIns}(3,4,5) P_{3}$, PtdIns (4,5) $P_{2}$ and phosphatidic acid [4]. In this study, we found no effects of these phospholipids on AtPDK1 autophosphorylation or AtS6k2 phosphorylation (Fig. 8), which is in agreement with the activation of S6K by mammalian PDK1 [1]. The present consensus of the mechanism behind 3-phosphoinositide-dependent activation of PKB by PDK1 is that binding of the lipid species to the PDK1 PH domain does not directly affect the activity of the PDK1 kinase domain $[1,31]$. However, in vivo, lipid binding localises PDK1 to the plasma membrane where it can interact with and activate PKB, also recruited to the plasma membrane through a PtdIns $(3,4,5) P_{3}$-binding PH domain [32,33]. In contrast, PDK1 activation of other substrates, such as SGK and S6K, lacking PH domains, is not dependent on 3-phosphoinositides [1]. Instead, the phosphorylation of $\mathrm{S} 6 \mathrm{~K}$ is regulated by a hydrophobic motif in the substrate [34]. Recently, AtPDK1 activity towards the N-terminal kinase domain of murine RSK2 was seen to increase in the presence of such a motif [35]. Since neither phosphatidic acid nor $\operatorname{PtdIns}(4,5) P_{2}$ affected AtS6k2 phosphorylation, we suspect that activation of AtS6k2 is regulated in a way distinct from the AtPDK1ACG1 pathway, which was recently reported to regulate root hair formation in A. thaliana and to involve phosphatidic acid [6].

We are still in the beginning of understanding the role of the plant PDK1. We have now shown that AtPDK1, like its mammalian counterpart, needs autophosphorylation at several sites for full downstream phosphorylation activity. In mammalian PDK1 most of the in vitro autophosphorylation sites identified have also been identified in vivo in cell lines transiently expressing the protein [13-15]. The phosphorylation level of the different sites may, however, differ between PDK1s analysed as E. coli recombinant proteins versus PDK1s transiently expressed in vivo. Identification of phosphorylation sites in the recombinant AtPDK1 using mass spectrometry combined with mutational analysis of identified sites and of sites suspected to be of importance for activity revealed clear differences in phosphorylation sites, and in their respective roles in rendering the kinase active and functional, between the plant and mammalian enzyme. Apart from identifying natural PDK1 substrates and regulatory proteins in plant cells it is important to corroborate the role of the different phosphorylation sites under more in vivo-like situations, e.g. using transient expression of PDK1 in protoplasts.

\section{Acknowledgements}

We thank Adine Karlsson for excellent technical assistance.

We thank Drs. Maria Deak (Department of Biochemistry, University of Dundee, UK), K. Shinozaki (RIKEN, Ibaraki, Japan) and Robert J. Ferl (Biotechnology and Horticultural Sciences, University of Florida, USA) for the generous gifts of the AtPDK1 clone, AtS6k2 and 14-3-3 cDNAs respectively.

This work was supported by the German plant genome project GABI (BMBF Project 32P2244) and the Max Planck Society, The Carl Trygger Foundation, The Crafoord Foundation, The Swedish Council for Environment, Agricultural Sciences and Spatial Planning, The Swedish Foundation for Strategic Research, and The Swedish Research Council. 


\section{References}

[1] A. Mora, D. Komander, D.M.F. van Aalten, D.R. Alessi, PDK1, the master regulator of AGC kinase signal transduction, Semin. Cell Dev. Biol. 15 (2004) 161-170.

[2] P. Storz, A. Toker, 3'-Phosphoinositide-dependent kinase-1 (PDK-1) in PI 3-kinase signaling, Front. Biosci. 7 (2002) 886-902.

[3] A. Dufner, G. Thomas, Ribosomal S6 kinase signaling and the control of translation, Exp. Cell Res. 253 (1999) 100-109.

[4] M. Deak, A. Casamayor, R.A. Currie, P. Downes, D. Alessi, Characterisation of a plant 3-phosphoinositide-dependent protein kinase1 homologue which contains a pleckstrin homology domain, FEBS Lett. 451 (1999) 220-226.

[5] L. Bögre, L. Ökrész, R. Henriques, R.G. Anthony, Growth signalling pathways in Arabidopsis and the AGC protein kinases, Trends Plant Sci. 8 (2003) 424-431.

[6] R.G. Anthony, R. Henriques, R. Helfer, T. Mészáros, G. Rios, C. Testerink, T. Munnik, M. Deak, C. Koncz, L. Bögre, A protein kinase target of a PDK1 signalling pathway is involved in root hair growth in Arabidopsis, EMBO J. 23 (2004) 572-581.

[7] T. Mizoguchi, N. Hayashida, K. Yamaguchi-Shinozaki, H. Kamada, K. Shinozaki, Two genes that encode ribosomal-protein S6 kinase homologues are induced by cold or salinity stress in Arabidopsis thaliana, FEBS Lett. 358 (1995) 199-204.

[8] F. Turck, S.C. Kozma, G. Thomas, F. Nagy, A heat-sensitive Arabidopsis thaliana kinase substitutes for human $\mathrm{p} 70^{\mathrm{s} 6 \mathrm{k}}$ function in vivo, Mol. Cell. Biol. 18 (1998) 2038-2044.

[9] J.W. Han, P.B. Pearson, P.B. Dennis, G. Thomas, Rapamycin, wortmannin and the methylxanthine SQ20006 inactivate $\mathrm{p} 70^{\mathrm{s} 6 \mathrm{k}}$ by inducing dephosphorylation of a novel set of sites, J. Biol. Chem. 270 (1995) 21396-21403.

[10] F. Turck, F. Zilbermann, S.C. Kozma, G. Thomas, F. Nagy, Phytohormones participate in an S6 kinase signal transduction pathway in Arabidopsis, Plant Physiol. 134 (2004) 1527-1535.

[11] H.R. Reyes de la Cruz, R. Aguilar, E. Sanches de Jimenez, Functional characterization of a maize ribosomal S6 protein kinase (ZmS6K), a plant ortholog of metazoan p70(S6K), Biochemistry 43 (2004) 533539.

[12] M.J. Wick, F.J. Ramos, H. Chen, M.J. Quon, L.Q. Dong, F. Liu, Mouse 3-phosphoinositide-dependent protein kinase-1 undergoes dimerization and trans-phosphorylation in the activation loop, J. Biol. Chem. 278 (2003) 42913-42919.

[13] A. Casamayor, N.A. Morrice, D.R. Alessi, Phosphorylation of Ser241 is essential for the activity of 3-phosphoinositide-dependent protein kinase-1: identification of five sites of phosphorylation in vivo, Biochem. J. 342 (1999) 287-292.

[14] J.M. Wick, K.R. Wick, H. Chen, H. He, L.Q. Dong, M.J. Quon, F. Liu, Substitution of the autophosphorylation site $\mathrm{Thr}^{516}$ with a negatively charged residue confers constitutive activity to mouse 3-phosphoinositide-dependent protein kinase-1 in cells, J. Biol. Chem. 277 (2002) 16632-16638.

[15] J. Park, M.M. Hill, D. Hess, D.P. Brazil, J. Hofsteenge, B.A. Hemmings, Identification of tyrosine phosphorylation sites on 3-phosphoinositide-dependent protein kinase-1 and their role in regulating kinase activity, J. Biol. Chem. 276 (2001) 37459-37471.

[16] S. Grillo, T. Grémeaux, A. Casamayor, D.R. Alessi, Y.L. MarchandBrustel, J.F. Tanti, Peroxovanadate induces tyrosine phosphorylation of phosphoinositide-dependent protein kinase-1, Eur. J. Biochem. 267 (2000) 6642-6649.

[17] L.Q. Dong, F.J. Ramos, M.J. Wick, M.A. Lim, Z. Guo, R. Strong, A. Richardson, F. Liu, Cloning and characterization of a testis and brain-specific isoform of mouse 3'-phosphoinositide-dependent protein kinase-1, mPDK-1B, Biochem. Biophys. Res. Commun. 294 (2002) 136-144.
[18] M.J. Wick, L.Q. Dong, R.A. Riojas, F.J. Ramos, F. Liu, Mechanism of phosphorylation of protein kinase B/Akt by a constitutively active 3-phosphoinositide-dependent protein kinase-1, J. Biol. Chem. 275 (2000) 40400-40406.

[19] A. Balendran, A. Casamayor, M. Deak, A. Paterson, P. Gaffney, R. Currie, C.P. Downes, D.R. Alessi, PDK1 acquires PDK2 activity in the presence of a synthetic peptide derived from the carboxyl terminus of PRK2, Curr. Biol. 9 (1999) 393-404.

[20] M. Frodin, C.J. Jensen, K. Merienne, S. Gammeltoft, A phosphoserine-regulated docking site in the protein kinase RSK2 that recruits and activates PDK1, EMBO J. 19 (2000) 2924-2934.

[21] N. Fujita, S. Sato, A. Ishida, T. Tsuruo, Involvement of Hsp90 in signalling and stability of 3-phosphoinositide dependent protein kinase-1, J. Biol. Chem. 277 (2002) 10346-10353.

[22] S. Sato, N. Fujita, T. Tsuruo, Regulation of kinase activity of 3-phosphoinositide-dependent protein kinase-1 by binding to 14-3-3, J. Biol. Chem. 277 (2002) 39360-39367.

[23] P.J. Sehnke, M. Rosenquist, M. Alsterfjord, M. DeLille, M. Sommarin, C. Larsson, R.J. Ferl, Evolution and isoform specificity of plant 14-3-3 proteins, Plant Mol. Biol. 50 (2002) 1011-1018.

[24] F. Svennelid, A. Olsson, M. Piotrowski, M. Rosenquist, C. Ottman, C. Larsson, C. Oecking, M. Sommarin, Phosphorylation of Thr-948 at the $\mathrm{C}$ terminus of the plasma membrane $\mathrm{H}^{+}$-ATPase creates a binding site for the regulatory 14-3-3 protein, Plant Cell 11 (1999) 2379-2391.

[25] J.C. Bearden Jr., Quantification of submicrogram quantities of proteins by an improved protein-dye binding assay, Biochim. Biophys. Acta 533 (1978) 525-529.

[26] U.K. Laemmli, Cleavage of structural proteins during the assembly of the head of bacteriophage T4, Nature 227 (1970) 680-685.

[27] M.P. Molloy, P.C. Andrews, Phosphopeptide derivatization signatures to identify serine and threonine phosphorylated peptides by mass spectrometry, Anal. Chem. 73 (2001) 5387-5394.

[28] H. Chen, F.H. Nystrom, L.Q. Dong, Y. Li, S. Song, F. Liu, M.J. Quon, Insulin stimulates increased catalytic activity of phosphoinositidedependent kinase-1 by a phosphorylation-dependent mechanism, Biochemistry 40 (2001) 11851-11859.

[29] M. Rosenquist, M. Alsterfjord, C. Larsson, M. Sommarin, Data mining the Arabidopsis genome reveals fifteen 14-3-3 genes. Expression is demonstrated for two out of five novel genes, Plant Physiol. 127 (2001) 142-149.

[30] M. Alsterfjord, P.C. Sehnke, A. Arkell, H. Larsson, F. Svennelid, M. Rosenquist, R.J. Ferl, M. Sommarin, C. Larsson, Plasma membrane $\mathrm{H}^{+}$-ATPase and 14-3-3 isoforms of Arabidopsis leaves: Evidence for isoform specificity in the $14-3-3 / \mathrm{H}^{+}$-ATPase interaction, Plant Cell Physiol. 45 (2004) 1202-1210.

[31] B. Vanhaesebroeck, D.R. Alessi, The PI3K-PDK1 connection: more than just a road to PKB, Biochem. J. 346 (2000) 561-576.

[32] R.A. Currie, K.S. Walker, A. Gray, M. Deak, A. Casamayor, P. Downes, P. Cohen, D.R. Alessi, J. Lucocq, Role of Phosphatidylinositol 3,4,5-trisphosphate in regulating the activity and localization of 3-phosphoinositide-dependent protein kinase-1, Biochem. J. 337 (1999) 575-583.

[33] L. Stephens, K. Anderson, D. Stokoe, H. Erdjument-Bromage, G.F. Painter, A.B. Holmes, P.R.J. Gaffney, C.B. Reese, F. McCormick, P. Tempst, J. Coadwell, P.T. Hawkins, Protein kinase B kinases that mediate phosphatidylinositol 3,4,5-trisphosphate-dependent activation of protein kinase B, Science 279 (1998) 710-714.

[34] R.M. Biondi, A. Kieloch, M. Currie, M. Deak, D.R. Alessi, The PIF-binding pocket in PDK1 is essential for activation of S6K and SGK, but not PKB, EMBO J. 20 (2001) 4380-4390.

[35] J. Silber, T.L. Antal, S. Gammeltoft, T.E. Rasmussen, Phosphoinositide-dependent kinase-1 orthologues from five eukaryotes are activated by the hydrophobic motif in AGC kinases, Biochem. Biophys. Res. Commun. 321 (2004) 823-827. 